\title{
"So many extra safety layers:" Virtual service provision and implementing social distancing in interpersonal violence service agencies during COVID-19
}

\author{
Rachel J. Voth Schrag ${ }^{1}$ (I) $\cdot$ Sarah Leat ${ }^{2} \cdot$ Bethany Backes $^{3} \cdot$ Saltanat Childress $^{4} \cdot{\text { Leila } \text { Wood }^{5}}^{5}$
}

Accepted: 14 December 2021 / Published online: 28 January 2022

(c) The Author(s), under exclusive licence to Springer Science+Business Media, LLC, part of Springer Nature 2021

\begin{abstract}
The coronavirus pandemic necessitated rapid shifts in approach for service providers working with survivors of interpersonal violence. To reduce the spread of the virus, providers and agencies implemented a rapid and unplanned expansion of virtual services while also developing new protocols to support safe and socially distant in-person services. To understand how these shifts have impacted victim service professionals and the survivors they serve, to provide guidance for on-going efforts, and to inform planning for future public health emergencies, this study asks the question: What approaches did the interpersonal violence workforce use to address social distancing needs during COVID-19? Semi-structured interviews were conducted from July to December 2020 with 33 interpersonal violence service providers from across the United States, and data were analyzed via conventional content analysis with additional steps for data credibility. Findings fall within two primary categories: 1) Technology and Virtual Service Provision; and 2) Social Distancing for In-person Services. Within each category, a number of themes emerged illustrating strengths and challenges of each approach, and the complex web of technological, safety, and public health considerations being balanced in interpersonal violence service agencies. These results provide guidance for the implementation of virtual services in an on-going manner, as well as underscoring the importance of future planning to facilitate effective in-person but physically distant services. There is also a clear need for agencies to support the interpersonal violence workforce to reduce occupational stress and enhance skills and capacities with new forms of services.
\end{abstract}

Key Words Intimate Partner Violence · Sexual Assault · Virtual Services · Telehealth · COVID-19

Rachel J. Voth Schrag

Rachel.vothschrag@uta.edu

Sarah Leat

srrbnsn2@memphis.edu

Bethany Backes

Bethany.Backes@ucf.edu

Saltanat Childress

Saltanat.childress@uta.edu

Leila Wood

leiwood@utmb.edu

1 University of Texas at Arlington School of Social Work, 211

S. Cooper, Arlington, TX 76016, USA

2 University of Memphis, Memphis, TN, USA

3 University of Central Florida, Orlando, FL, USA

4 University of Texas at Arlington, Arlington, TX, USA

5 University of Texas Medical Branch, Galveston, TX, USA
Decreased economic stability, increased family stress, prolonged exposure to abusive partners, and reductions in services capacity, among other factors have all been cited as contributors to an estimated $8 \%$ increase in intimate partner violence (IPV) early in the coronavirus pandemic (McClay, 2021; Nnawulezi \& Hacskaylo, 2021; Piquero et al., 2020; Piquero et al., 2021; Wood, Baumler, et al., 2021a). The pandemic and resulting stay-at-home orders, which began in March 2020 in the United States, required interpersonal violence service providers (such as intimate partner violence shelters and rape crisis centers) to adopt new intervention frameworks and modalities (Wood et al., 2020a). These changes included quickly shifting to virtual services and implementing strategies to facilitate socially distanced in-person services (Kaukinen, 2020). While implementing these adaptations, service providers faced significant strain as first responders to an evolving crisis. To understand the changes being implemented within the service sector and provide evidence to inform future public health responses, 
this study explores the experiences of interpersonal violence service providers implementing virtual services and social distancing guidelines during the first year of the COVID-19 pandemic response in the United States.

\section{Background}

The Centers for Disease Control and Prevention estimate that 81 million Americans will experience sexual violence, physical violence, and/or stalking by an intimate partner during their lifetime (Breiding et al., 2014). Experiencing interpersonal violence creates a wide range of serious physical and mental health impacts for survivors and their families, as well as short and long term economic, educational, and intergenerational consequences (Castro et al., 2017: Chisholm et al., 2017; Postmus et al., 2012; Sedziaofa et al., 2016; Soleimani et al., 2017; Valera \& Kucyi, 2017). Survivors of interpersonal violence, including intimate partner violence (IPV), sexual assault (SA), and child abuse and neglect $(C A \& N)$ engage in support provided by a network of community-based service agencies. These agencies provide a wide range of survivor-centered services, including advocacy, counseling for children and adults, housing, legal, and economic supports, all focused on the needs and goals of the individual survivor and their family (Macy et al., 2009; NNEDV, 2021; Sullivan \& Goodman, 2019; Wilson et al., 2015). Guided by these tenets, community-based agencies seek to provide voluntary services aiming to facilitate survivor-defined participation in formal services as needed (Nnawelezi et al., 2018; Wilson et al., 2015). These services have been demonstrated to reduce the risk of future violence and increase well-being, emphasizing survivor goals within a trauma-informed and culturally relevant approach (Nnawelezi et al., 2018; Rivas et al., 2019).

\section{Social Distancing During COVID-19}

In the United States, interpersonal violence service providers were faced with rapidly implementing social distancing strategies to reduce contact between infected and noninfected persons in order to stop disease transmission, along with other interventions to control the spread of COVID19 , with most jurisdictions beginning the implementation of these practices in March of 2020 (USHHS, 2020). Public health guidance focused on effective strategies to stop and reduce the spread of infectious droplets, particularly indoors and in communal settings. These included physical distancing, staying away from others when sick, isolation and quarantining of infected cases and close contacts, and the use of appropriate Personal Protective Equipment (PPE; e.g., face masks and shields) (Collignon, 2021). Initially, guidelines evolved quickly as more was learned about the transmission of COVID-19 (Badr et al., 2020), and ultimately a wide range of measures were put in place across settings, with government agencies highlighting the need to use strategies in combination to reduce risk and control disease spread (Collignon, 2021). For interpersonal violence service providers, this often led to the need to make difficult decisions, such as reducing service capacity or closing key services, and the need to continually review and shift agency policies and procedures (Wood et al., 2020). In particular, emergency shelter for IPV survivors has long been a critical, life-saving service and front door for accessing other services and supports. This necessarily in-person support has frequently been offered in formats that are not easily aligned with social distancing expectations, including having multiple families share rooms or bathrooms, communal living spaces, and joint meals. Evidence from service-providers suggests that adjustments to shelter capacity, format, and processes were among the largest initial challenges to adjusting to social distancing guidance (Wood et al., 2020).

\section{Virtual Services}

In the years prior to the pandemic, virtual services were already gaining attention as an additional tool for victim service providers, as the proliferation of internet and smartphone use changed the way people communicate and build relationships (Merolli et al., 2014; Voth Schrag et al., 2021). With the service sector's focus on providing individualized services built around the preferences and needs of each survivor, agencies had begun to build virtual services, such as chat and text-based crisis hotlines, to allow individuals to engage with services using modes of communication most comfortable for them (Nesmith, 2018; Wood, Hairston, et al., 2021b). Initial data suggest that these services can fill an important gap. For example, an outcomes assessment of a youth crisis-text hotline found that $65 \%$ of users had positive outcomes (Nesmith, 2018). Further, there is evidence that virtual services may have specific benefits in terms of reducing barriers to service entry, with evidence of a "disinhibition effect" reducing the perceived stigma of disclosure, allowing for greater engagement, particularly among youth (Budinger et al., 2015).

However, due to a combination of factors, including concerns over the potential safety risks created by digital surveillance by an abusive partner, the cost of technology infrastructure, and an already stretched-thin workforce, the interpersonal violence services field had not widely adopted virtual services prior to the Coronavirus pandemic (Brignone \& Edleson, 2019). Indeed, quantitative research with providers in the first months of the pandemic indicate that under $10 \%$ of service providers were using video conferencing technologies prior to March 2020, and that this increased to nearly $60 \%$ by July 2020 (Wood, Baumler, 
et al., 2021a). This research similarly demonstrates a $15 \%$ increase in texting with clients, an $18 \%$ increase in computer chat, and a $12 \%$ increase in facetiming with clients over the same period (Wood, Baumler, et al., 2021a). Such significant shifts in services would typically occur over much longer timeframes, and with substantial financial outlay in terms of staff training, hardware, software, and evaluative research, yet the context of the pandemic made this impossible. Further, some forms of virtual services which rely on text rather than voice-based communication (e.g., chat, text, and e-mail) also provide a potentially safer way to reach out for survivors who are in close proximity to an abusive or unsupportive individual, making them uniquely applicable in the context of COVID-19 social distancing and stay-at-home orders (Wood, Baumler, et al., 2021a).

\section{Current Study}

The coronavirus pandemic, and need to implement social distancing guidance while maintaining service access in the face of escalating violence, created a perfect storm for the rapid expansion of virtual services within interpersonal violence service agencies, in concert with new protocols to support safe and socially distant in-person services. To capture these rapid changes, this study asks the question: What approaches did the interpersonal violence workforce use to address social distancing needs during COVID-19? It seeks to understand the strategies that evolved within community-based interpersonal violence services to meet public health guidance related to social distancing in the wake of the COVID-19 pandemic, as well as the experiences of the anti-violence service professionals faced with implementing those service adaptations.

\section{Methods}

The data for this study were collected as part of a mixedmethods project which documented the experiences of interpersonal violence survivors and service providers from April to December 2020 in the United States. The project included an initial quantitative survey with nearly 500 survivors and service providers fielded from April to June 2020, which aimed to capture a quick snapshot of safety and service experiences during the initial phase of the COVID response (see Wood, Baumler et al., 2021a; Wood et al., 2020 for detailed study information). With partnerships from national IPV and SA advocacy agencies, such as the National Coalition Against Domestic Violence, as well as regional partners including the Texas Council on Family Violence, the Texas Association Against Sexual Assault, and professional networks across several states, participants were recruited into the initial web-based survey via social media posts and professional list-serve distributions. The survey concluded by asking if participants were willing to be re-contacted for a semi-structured interview. The data for the current study come from those interviews with $(n=33)$ service providers from across the United States, which were conducted from July to December 2020.

\section{Procedure}

Interviews were conducted via ZOOM video conference or over the phone based on the preference of the participant. Interviews lasted between 35 and 90 minutes and were professionally transcribed. All interviews were conducted by study team members who have previous experience with both qualitative interviewing and victims service provision. Prior to the outset of data collection, study procedures were approved by the institutional review board of the sponsoring university. All participants provided verbal consent and permission for audio recording prior to the beginning of data collection, and participants received a $\$ 25$ gift card.

The interview protocol queried participant demographics, personal experiences with the pandemic, client safety and service use during the pandemic, as well as agency approaches to social distancing and health guidance, and occupational stress. Example questions that elicited data related to technology use or social distancing adaptations included the following: How has your job changed during the Coronavirus Pandemic? and How has your use of technology for work with clients changed during the Coronavirus Pandemic?

\section{Participants}

Service providers $(n=33)$ in intimate partner violence, sexual assault, child abuse, or human trafficking focused community-based agencies participated in semi-structured interviews. Inclusion criteria included being at least 18 years of age, being able to complete an interview in English or Spanish, and working in a paid role in advocacy, direct client service, or agency leadership in community-based victim serving agencies during the first phase of the COVID-19 pandemic response in the United States (March-July 2020). Participants ranged in age from 28 to 65 , and most identified as female, with others identifying as male or non-binary. Twenty participants identified as White, with six identifying as Hispanic/Latinx and others identifying as Black or Asian. Participants described their job tasks as including survivor advocacy, housing services, legal services, prevention education, and agency leadership. Participants came from across the United States, with the plurality from the state of Texas. 


\section{Results}

The data reflected two primary categories of service adaptations: 1). Technology and Virtual Service Provision; and 2) Social Distancing for In-person Services. A visual depiction of key categories and findings is in Table 1. Within each category, a number of themes emerged illustrating the complex web of technological, safety, and public health considerations being balanced by service providers in interpersonal violence service agencies. <table 1 about here>

\section{Technology and Virtual Service Provision}

Perhaps the most rapid adaptation in victim services at the outset of the COVID-19 pandemic response was the shift towards virtual services in a field which previously largely avoided such modalities. Advocates rapidly implemented ZOOM based visits, began using e-mail and texting with survivors more frequently, and continued to rely on the phone to accomplish many of their work tasks. These shifts often were accompanied by increased focus on emergent or crisis needs- in-the-moment advocacy, and more frequent but shorter service 'touches,' with several e-mails or texts in a week taking the place of a twice-monthly in-person visit, for example. Unsurprisingly, service providers had a great deal to say about this rapid change in their service delivery medium, with both positive and negative experiences and views on integrating virtual services into their post-pandemic work.

Benefits of virtual services Across interviews, a set of positive impacts and outcomes of the quick shift towards virtual services were frequently cited, with many service providers reflecting that increased accessibility and added privacy led to disclosures and help-seeking from survivors who previously may not have felt safe or comfortable with services.
Many service providers identified that, for a large segment of their clientele, removing the need to physically travel to an agency to receive services proved a significant reduction in the burden of service access. Without transportation, child care, or scheduling conflicts, services became more accessible to parents, full-time workers, those with mobility limitations, and survivors who might otherwise fear questions from an abusive partner about their travel. As one IPV service provider noted: "I think a big benefit is the convenience for clients that they are able to do the services from home. I think that takes away that barrier of a $30 \mathrm{~min}$ ute drive or a bus ride." A sexual assault service provider noted: "It's easier on their schedules to be able to meet with me because they don't have to factor in travel time and... when they have to work, that kind of thing." This advantage seemed particularly salient for providers who worked in crisis response roles, where chat and text help lines expanded rapidly. The combination of the need for immediate support in crisis situations with an increasing acceptability of digital communication in society created what one agency leader described as a 'perfect storm' to rapidly advance virtual services where they had previously been avoided. As another agency leader noted:

The text help line, that took off. That was a real hit. We did a little bit of advertisement on the front end. We've had almost immediate engagement with that service. I think just society was going that way anyway. People feel more comfortable texting than they do verbal communication. I think even if we had introduced five years ago, ten years ago, it would've been a hit then too. I just think it was really time.

Along with the immediacy that virtual services can provide, advocates pointed to other ways these platforms can break down accessibility barriers for diverse populations. One provider who works in a program that serves a large number of immigrant and refugee survivors noted that the
Table 1 Key Findings: Virtual service provision and social distancing during COVID-19

\begin{tabular}{ll}
\hline Technology \& Virtual Services & Social Distancing for In-Person Services \\
\hline Benefits of moving online: & Social Distancing in Communal Living: \\
- Accessibility (transportation, child care) & $\bullet$ Balancing safety from violence \& \\
- Immediacy of services & health \\
- Language access & - Isolation and control in shelters mim- \\
- Anonymity \& comfort disclosing & icking abusive dynamics \\
& - Reduced capacity \\
Challenges of moving online: & Challenges with in-person services \\
- Empathic connection is harder & - Frequent shifts in procedures \\
- Assessing environment is challenging & - Altering Spaces to increase safety \\
- Technological challenges & - Access to PPE \& cleaning supplies \\
- Boundary maintenance & - Shifts in who is 'in the office' \\
- Technology Safety is part of the job now & $\bullet$ Tensions between work-form-home \\
& and site-based staff \\
& - Difficulty with masks and physical \\
& distance for in-person connection \\
\hline
\end{tabular}


broader societal move towards digital and text-based services has allowed them to connect with a larger number of survivors using translation services. Even for providers who speak multiple languages, they sometimes work with clients in languages in which they are not fluent. They noted that "I have a lot of non-English speaking [clients] right now. I probably have five that do not speak English at all. Via text, it's okay. I can Google translate in text." Another advocate talked about being able to use simultaneous translation through a language line provider while on ZOOM, allowing her to interact with survivors more effectively than previous three-way calling strategies. Survivors who speak languages other than English are not the only groups who advocates identified as being uniquely more accessible. Service providers who work in university settings also repeatedly and specifically identified the ways that shifting service modes created increased engagement with their clients. One advocate shared that "I think it's easier on their schedules to be able to meet with me because they don't have to factor in travel time and when they're going to be on campus or not and when they have to work." Further, for some campus based survivors, the risk of being seen entering the victim service office was a barrier, while others did not frequently come to campus, and thus did not have access to on-campus services as readily. As one Campus-based advocate shared:

I definitely want to keep, um, the ability to have the video connection. Cause I think I also realized we weren't accessible for some students and students couldn't kind of meet in person for whatever reason. And so to not have even offered this in the past now feels like such a huge oversight.

Along with increasing access, service providers identified ways in which the transition to virtual services enhanced the perceived acceptability of seeking services for some survivors, reducing barriers to entry for folks who previously may have been uncomfortable seeking services. The increased anonymity that digital communication provides was seen as a benefit in many cases. As one agency leader shared:

We've been noticing an increase in male survivors when it comes to our workshops, or support groups, or counseling. More male survivors are ... I don't know if it's the stigma of actually being present and the public seeing it's a male survivor. They could just kind of hide ... Not hide, but just be behind a screen. That's one thing.

This was echoed by a community prevention worker, who noted the usefulness of anonymity in allowing folks to reach out, particularly via virtual routes that don't require the survivor to disclose their identity to the service worker, saying "Yeah. In some of the trainings that I do I've been using the chat functions for people to message me privately and ask anonymous questions. That has been great. Or just reach out and form contact that way."

Challenges presented by virtual services While most of the service providers interviewed had a neutral to positive overall outlook on the pandemic-facilitated expansion of virtual services, they nonetheless highlighted a wide range of challenges, some of which are inherent to the virtual medium and some unique to the challenges of adapting to new work styles in the midst of a global public health crisis.

Difficulty Connecting The challenges of connection in virtual work was frequently mentioned, although it emerged in different ways with different service providers. For many in client-facing roles, a key point of challenge was developing authentic emotional connections over a virtual platform, while others felt unable to engage in important parts of their job virtually, and others highlighted literal challenges with connection- an inability to contact clients, or challenges with technology once a contact has been made. Several participants reflected on the new challenges of building strong and therapeutic relationships in virtual environments. As one advocate shared, "I think the biggest stress is not being able to see the client. And a lot of misunderstandings can happen." Even when there is a strong bond between service provider and survivor, virtual environments create additional challenges. For students and survivors whose lives also shifted predominantly online in the wake of the pandemic, another screen was often not what they wanted. As one advocate noted, "A lot of us struggle to get the clients online, especially with minors...it's even harder. They're already on the computer all day. So then they will have an appointment at five o'clock with a case manager and forget it." Also reflecting on the challenges connecting with children, one participant shared:

I feel like I've gotten more gimmicky because I work in child welfare and it's harder to get younger children to stay engaged in a video call than an in-person interaction... [I] would spend a lot of time and energy, trying to find ways to help kids actually feel engaged and not like just zone out.

This difficulty with connection virtually was further compounded by challenges with specific tasks that some anti-violence service providers need to perform, like child advocates who assess the safety and well-being of children during in-person home visits. One advocate gave a number of examples unique to the challenges of doing virtual safety checks with children:

The ability to have private discussions has been really altered, especially with young kids who need to have the phone held up. And then it's very easy for parents 
to just show part of the child and we don't get a good sense of if there's any bruising or any marks that we should be aware of..... And then I also had a situation a few weeks ago where I did an unannounced call into a visit with a mom and her children to try to see if there was anyone else at the visit because we'd had concerns of that. And there was nobody there, but then come to find later on the children said there was someone there and mom just didn't show the camera.

The challenges assessing what is going on outside of the small area of camera view was echoed by another child welfare worker, who shared: It has gotten more difficult to feel like I have an accurate sense of the home environment that the child is in because I can't physically go into the home and to see their caregivers in-person. For service providers working with adult survivors, this same challenge took on a different form, with fear related to who might be listening into a session or tracking a participants movements electronically.

As ever, service providers had a wide range of strategies and work arounds that they attempted to address these challenges with connection. For assessing the safety of a child's environment, one worker noted that: [She] might ask them to show me things. Or if they're talking about a meal that they have, I might have, well, like I don't know, I'd have to see different parts of their home.

While there are specific challenges for children's services, services providers also noted the difficulty of connecting with adult clients, partly brought on by the challenges of connecting via phone, especially from a protected or blocked number used by many service providers. As one provider noted:

And one of the frustrating things is I emailed them and tell them, "I'm going to call you from a blocked number at the time of your appointment. Answer the phone." And they haven't been answering the phone, I think one person answered...And even the number that they see is a fake number. So even if you redial it, it'll say, "I'm sorry. This phone is not in service." So I also have to tell them that too is, "Look, that phone number that popped up, that's not how you reach us. This is my number. Here's my email."

Participants also felt that, with survivors who had limited access to technological mediums beyond the phone (for example, ZOOM or computer-based chat with stable internet or cellular data) access has been more limited than ever. This further exacerbated disparities in service access based on economic stability and geographic region. This not only limited the venues in which services are accessible, but makes many of the activities that advocates complete with survivors more challenging. For some, the limitations of technology meant that working with documents or legal advocacy that involves filling out forms or reviewing materials posed a specific challenge. As one advocate shared:

For many or most of my clients... all they have is their phone. Still that's not enough because it's hard to do... Accessing the internet and the hearings on zoom and etc. It's difficult to do on a phone, right, and be switching back and forth to documents or things like that that they need. The technology access has been really difficult for many.

Boundaries The challenge of virtual connection takes on unique contours for service providers who engage in traumafocused work with survivors of violence. For those who have roles as counselors or therapists in particular, the boundaries they draw around their work-life shifted significantly as work 'came home.' As one counselor noted, "Boundaries are my favorite thing ever. So I, at first even struggled so much with like, I don't want people to see a wall of my house that felt like this is weird." Both the advantages of and the challenges posed by this new intimacy- being in each other's space- were echoed frequently, as advocates and counselors felt some level of invasion of their work into their personal space, but noting that survivors sometimes felt more comfortable being in their own 'safe' spaces. Service providers gave many examples of how they worked to develop a therapeutic virtual environment, including thinking about digital backgrounds, providing music or other sound cues, and using verbal check ins to help clients make sure their own environments were as comfortable as possible. An advocate who did many virtual trauma-focused visits early in the pandemic shared:

They get to be in their house and they maybe have some of the things that help them cope or feel safe at their house. Like maybe, they can be with their pets during their session or they have more coping skills available right then and there. We've noticed for some that it's a positive thing because they're already somewhere they're really comfortable. There's actually clients where it felt almost like they do feel more comfortable to talk and to open up.

While another advocate also echoed the role of being able to be at home, having access to pets and enhanced therapeutic connection:

There's interestingly some aspects of the more therapeutic relationship that are opened up by Zoom in the sense that I see their rooms when they talk to me so we can get to know each other- that way or their 
pets come up to them and I can integrate those into a conversation.

Still, there are aspects of trauma work that are subtle but important, which service providers felt were lacking in their digital environments. One counselor noted:

There are a lot of...tricks that you could do with clients. You have some water available. You try and arrange your office in a certain way, have the lighting a certain way, maybe have some candy or something available. There were a lot of things you could do to improve the trauma-informed-ness of your practice that you can't do over Zoom.

Tech Safety as Part of the Work Now The emergence of virtual services in an increasingly technology mediated world led to changes in not only the platform of service provision, but the content of those services as well. Ensuring on-going safety, and educating survivors about the risks of virtual services, became the first thing service providers needed to attend to at the outset of every interaction. Checking in about a survivors' current safety, as well as coaching about digital surveillance and developing a safety plan in case things changed mid-visit, became the new "how are you feeling today" as a way of opening any session. As one advocate articulated:

I mean the biggest change right away. [Technology and safety] is just like the first thing we're talking about. Normally they'd be in my office so I could see that and know like we're in the same space and abusers are not here. I don't know that if someone's video chats and they're called, so literally the very first thing we're saying and talking about it, I'm wondering if you're safe right now. I'm wondering if, uh, someone could walk in and if so, what are plan? Are we going to completely end the chat in my, your friend from math class? And we have one professor to get it like right away, what's in safety plan. Will the students want this other person know who I am from campus? I know you're accessing resources. Is there a different, you know, could you go to a friend's house to talk to me that they won't see me?

\section{Social Distancing for In-person Services}

In victim serving agencies, the shift to heavy reliance on virtual services to promote social distancing during the pandemic response was complimented by extensive shifts in how in-person services were implemented. This created specific challenges for services that include communal living settings, and mandated significant shifts in cleaning and space planning, even as social distancing rules sometimes got pushed to the side when service providers felt they limited their ability to connect with survivors.

Creating social distance in communal living For many survivors of IPV, safe housing through emergency shelter is a critical 'front door' into services. Historically, shelter is often seen as the first step in a survivor's journey with services. This option, which often involves communal living, brought up new anxiety in the context of a pandemic, where living with a large group brings with it an increased risk of infection. An advocate in July 2020 shared:

There's only so much we can do to try to make them feel safe when there's something like a virus... For some of them, they've known their abuser for a decade or who knows how long. They think that they know, "Okay, I know what he's capable of, but this virus could kill me." So they think, at the time, making irrational decisions like victims do, staying with him may be safer than risking getting COVID in a shelter.

Another advocate had similar sentiments, while highlighting other potential factors influencing survivors' decisions about help seeking in the context of the pandemic, sharing in August 2020 that they knew of survivors saying: "How do I hedge my bets between possibly getting Coronavirus in the shelter or just that increased control that shelters have to put in place in order to prevent Coronavirus or hedge my bets with my abuser?" Indeed, several advocates talked about various new shelter policies and protocols, from enforced isolation upon entrance to heightened sanitation standards, to limitations on coming and going that in some ways replicated the experience of living in an abusive context. For many survivors, these stressors and restrictions mirrored the experience of living with an isolating or controlling partner, potentially adding another trauma trigger to deal with. Many service providers shared similar reflections to this advocate, who shared:

We're still on a stay home order with our shelter. We still have increasing cases, and so we monitor where they go and what they do. So if you're not working, you're not just going to go in and out all day. Sorry. You're just not. If you don't have an appointment, and if it's not medical or an emergent need, you're not going. I have to be the bad guy and say no. So when they can't leave property whenever they want to, they're like, "I feel like I'm right back with my abuser."

For some, the isolation that shelters implemented in order to reduce the risk of a COVID outbreak increased the difficulty of living in an already emotionally challenging environment. 
A victims' advocate working with military connected survivors shared in October 2020:

It's been harder for shelter reasons. A lot of my clients were already hesitant to go to a physical shelter, and then this just made that trying to convince somebody to go to a shelter even harder...I know them here and they were reassuring all the measures they were taking, and that they hadn't had any positive cases, and were updating us regularly, but still even with that it was really hard to commit somebody to take that step. Like I said, it's already hard enough, and that just make it even harder, but they reassured us that they had separate housing units and all of that, that they could access if they needed to if there was a family, or if somebody had been exposed and needed to be quarantined, or something to that effect. I've had a handful of clients need to go into hotels, or on base we have a transitional living facility. It's sort of like a hotel, but for military on base.

These concerns piled on top of the already significant challenges of living in shelter, and particularly created burdens for parents working to keep their children safe from both violence and illness, and for those children who are experiencing such significant life transitions. As shared by one agency leader:

Communal living is hard, it's miserable even in the best of scenarios, you add now I'm trying to keep you safe from a pandemic too, it just becomes a nightmare. I think that we're starting to see children come back into shelter, not young children but teenagers like 10 and up, 11 and up with their parents. Even if a parent understands it, staying in a single room by yourself with two, three kids, I just cannot even fathom that myself, and it's really hard for them. There are scheduled times for people to be in communal spaces, so we have a big backyard, and we have a kitchen and a dining room which are all open, but the playroom and the living room are still not open because there's not a really good way to keep them clean. They have scheduled times, and I don't know, I think that it's hard on everybody, but I think it's especially hard on kids who aren't fully functioning on the same level about the pandemic and the situation they're in overall to be in a shelter.

Evolving public health guidance meant frequent shifts in policies and procedures. For many shelter programs, initial steps included dramatically cutting shelter capacity to allow for greater space between residents, with many service providers echoing an advocate who shared that "we've reduced our shelter capacity so that everybody has their own bathroom, so that cut our rooms in half." While some providers talked about reduction in capacity, others noted that, in periods of the greatest community transmission, or at the pandemic outset, they stopped bringing survivors into shelter completely. One advocate shared: "They weren't letting anybody in. When the numbers started to spike, they were like, "Wait. We can't just let other people in because we don't know if you have the virus or not. So we can't jeopardize everybody else." Capacity was further limited in agencies who set aside specific quarantine rooms, as shared by an advocate:

So, we are normally a 40-bed shelter, and our capacity was cut in half because we have a lot of bunk beds. It's top and bottom bunk. That's not a six-foot distance, so we had to close all the top bunks to relate to social distancing. We had far less capacity than normal. Additionally, we had to close a bedroom entirely to retain it as a quarantine room

In the face of these limitations, agencies employed a wide range of creative solutions to house as many survivors as possible. Agencies worked with local hotels to increase the availability of short term hotel placements which could double as initial quarantine periods prior to shelter entry. Others developed new community partnerships to quickly increase access to transitional housing units in order to move folks through shelter more quickly and get families into their own units in which they could more effectively social-distance. Programs even took steps to re-work their outreach office space to provide additional space for shelter, or to deploy trailers or RVs on agency property to provide more safe, socially distanced housing.

Cleaning and Space adaptations Along with shifting housing capacities and rules limiting social interactions in shelter settings, agencies were adopting new cleaning strategies and implementing space adaptations in order to increase the safety of in-person services. New office features, like plexiglass, hand sanitizing stations, and distancing stickers emerged, as cleaning took on an added intensity for staff just as the volunteers who often help with those tasks were sent home. Residents and staff needed access to masks and other PPE, and agencies faced challenges accessing and affording cleaning supplies and PPE, especially early in the pandemic, as they were not considered essential health care services in many communities' pandemic response plans. As noted by one agency leader:

We were also really challenged with maintaining cleanliness to the $\mathrm{CDC}$ recommendations. That was a challenge because, of course, cleaning products were not available widely. So here when we're supposed to be as clean as possible, we couldn't get anything 
because we weren't a medical facility. At that stage, they were limiting quite a lot of things to medical providers only, and of course the local stores were out of many, many things.

Similarly, in an effort to reduce the number of people in a given space, many agencies cut the number of staff working in-person at any given time. This led to tension between "in-person" and "stay-at-home" workers. One agency leader described these tensions:

We also had a lot of dynamics between our outreach team, who we were able to convert to virtual appointments for survivors through doxy.me, or the Zoom for medical providers. Those kind of formats that we felt comfortable with, we moved a lot of them into those formats. Then we had our shelter folks who we can't provide virtual shelter services. I mean you're providing shelter services. So there began to be quite a rift between our outreach and shelter team with, "Oh, it's so stressful. I'm working from home and I've got the kids everywhere." That blend of how your personal and professional life, you used to be able to have a nice boundary and there's no boundary...Then you have your shelter advocates sitting through a staff meeting at the same time with those people having "the nerve" to complain about that when they wished they could be home, because they're here, and even though we're providing PPE and so forth, they're wondering, "What am I bringing home to my family?", and having that side of the coin to look at.

An additional contributor to these tensions was the increased workload for in-person folks needing to attend to physical plant issues along with their normal job duties. As one counselor noted, "We reduced drastically the amount of people in the office at a time, which has made the workload every day just more difficult for everyone."

In-person connection challenges For some service providers, particularly those with counseling or therapy job tasks, social distancing protocols posed challenges even where inperson services were allowed. Several providers noted that restrictions like distancing and mask wearing, particularly in one-on-one interactions, still felt inauthentic. Many providers highlighted how those protocols limited their ability to connect with survivors and engage in trauma focused or therapeutic work with the level of connection they would desire. As one provider shared, "I think that these masks have had a huge effect on our ability to interpret clients, whether it be literally understanding what they're saying or reading their emotions on their faces and noticing their facial expressions." She went on to note that "Even when you're in a situation like that where someone's trying to be vulnerable and express their worries and concerns, and there is a barrier like a mask between them, I think it's just created an awkwardness."

The impact of masks on connection was a frequent source of concern, with providers also raising issues with how they were experienced by survivors dealing with trauma, particularly those who might have previously experienced strangulation or sexual assaults in which breath was impeded. As one therapist shared, "We have to wear these masks. And I'm a therapist, and when they have emotions and they can't breathe because there's a mask over them, that's very frustrating and makes me not want to do it." Finding strategies to allow survivors to breath during trauma work became a task many advocates or therapists undertook, lobbying to allow survivors to use face shields or do therapy outside, because masks were experienced by some survivors as stifling and sometimes triggering.

Along with masking, many in-person therapeutic services moved out of carefully curated therapeutic office spaces into sterile rooms which allowed for greater physical distance between therapist and survivor. This adaptation was also frequently cited as impacting therapeutic quality. One therapist noted "We were supposed to do it six feet apart but it just felt so strange... We were probably more like three or something. After you develop relationships with folks it seems so odd to do it." Several participants shared about times when they felt strongly that being accessible and approachable (and thus, mask-less or physically close) was critical to the work they were doing with survivors- that the risk of illness was 'worth it' in some situations where survivors were in extreme distress. This attitude was more frequent among participants who felt less personal risk from COVID, while others who felt increased COVID risk expressed that the challenges of masking and distancing sometimes made 'ZOOM therapy' preferable for survivors dealing with trauma for whom feeling 'together but separate' due to COVID in-person restrictions was too emotionally fraught.

\section{Discussion}

Interpersonal violence victim service providers continued their efforts to provide critical, survivor-centered interventions throughout the COVID-19 pandemic, with frequent shifts in service modality, duration, location, and dynamics as public health guidance evolved. These interviews with 33 victim service providers highlight how advocates and survivors learned together what new risks and opportunities an increasingly digital world provides. Further, they describe the way victims services rapidly shifted to virtual and telehealth modalities, while in-person services became physically distant, but not always 'socially distant.' 


\section{Virtual Services}

For service providers, the primary benefit of expanded virtual services was their ability to be accessible to survivors. Removing barriers related to transportation and child care reduces the challenges of accessing services for survivors who juggle multiple roles, including managing safety and relationship challenges. Further, providers felt that digital services could facilitate language access in emergency situations where survivors may not otherwise have access to crisis care, and the ability to be anonymous (either actually anonymous to the provider, or simply unseen accessing services) was seen as a benefit to survivors who fear stigma related to service seeking, or fear that law or immigration enforcement could be called to an agency.

These findings point to important changes in the service delivery model for survivors of interpersonal violence, which could be maintained even as society emerges from some forms of social distancing. They suggest that, for agencies to continue to offer a virtual service model, service providers need to focus attention on their virtual front door. Steps which providers take to enhance survivor comfort in an in-person environment, such as providing water and snacks and creating a safe calm space for survivors to meet with advocates, should be replicated as much as possible in the digital environment, with additional attention to safety and digital security. This could include ensuring that advocates have a calming background, appropriate lighting, and a space free of background noise in order for survivors to feel comfortable and safe discussing their trauma, as well as encouraging survivors to create a comfortable space for themselves. In addition, ensuring that survivors are given clear instructions and ongoing support on the safe use of technology will be critical. Providing survivors with information about the selected virtual platform and suggestions for how to create a space for them to conduct video conference could assist advocates with being able to clearly communicate with survivors. This information could be provided via the agency website or in an initial intake session to allow for questions and answers and support survivors in implementing specific strategies.

In addition to creating a virtual front door, the findings of this study point to the importance of continuing virtual services and potentially adding them as part of an a la carte service model. The results revealed several benefits to offering virtual services, such as eliminating barriers regarding transportation, child-care, and lack of time to seek services. Offering a virtual option could allow more survivors to seek services in a more immediate fashion rather than having to wait for the opportunity to seek help. While there were challenges associated with connectivity and privacy, the results revealed that survivors' potentially feel safer attending services and accessing help online. Additionally, survivors who previously may have felt stigmatized for seeking services (such as male identified survivors), and those who do not want to be seen accessing interpersonal violence services in person, may feel more comfortable with the anonymity provided by virtual services. As such populations that are hard to reach for interpersonal violence service providers, or populations that experience marginalization may especially benefit from services expansion in this area.

Technology and internet access is critical for both survivors and advocates. During the pandemic when many advocates shifted to working exclusively in their homes, agencies realized quickly the technology needs and training associated with offering virtual services. Advocates needed encrypted laptops and tablets as well as a phone to call survivors from a protected number. In addition to providing advocates the opportunity to conduct services from their homes, the new technology allowed advocates to be flexible in connecting with clients. They could call survivors from multiple locations and connect with them from wherever they were. Allowing agencies to continue to use these resources, even if advocates return to offices, could open up more opportunities for advocates to connect with survivors in ways that meet survivors needs and eliminate barriers to help-seeking for survivors. Providing funding to train advocates on virtual platforms could also open doors for advocates and survivors to connect. These results also highlight to the importance of all community members having access to reliable wifi as a basic right. If virtual platforms are to continue to be a vital part of the service delivery models, advocacy for community access to internet and wifi is critical. The internet was a critical resource during the pandemic and it will continue to play a central role in the success of all community members. Providing advocates and survivors access to the internet by offering funding for hot spots is an important step to the success of a virtual service model. Similarly, providing consistent and high quality language access services in virtual environments will be crucial, as relying on tools like Google Translate and other limited strategies could further gaps in services for survivors who speak languages other than English.

The pandemic has accelerated the trend toward the use of virtual services, with accompanying implications for service providers responding to IPV. The trend towards institutionalizing and normalizing virtual services includes the expansion of virtual modalities, including chat and text-based hotlines, remote counseling, web-based electronic health platforms, and mobile health. Beyond these remote services, other innovative digital health practices, such as steppedcare models and provision of preventive and self-management services are on the horizon and may augment or even replace some traditional services (Taylor et al., 2020). However, the institutionalization of these virtual services creates both new policy implications for managing the quality, 
security, and safety of services. Taylor et al. (2020) present a partial roadmap of the systems- and policy-level requirements to address needs associated with training, licensing, safety, privacy, payment, and evaluation to institutionalize service delivery to promote use and access to promising interventions as a mainstream platform.

Agencies could look to the training approach outlined for nurses and midwives implementing IPV screening in telehealth for practical guidance in the implementation of IPV services in telehealth contexts (Jack et al., 2021). This example highlights the process of scaling up telehealth training and intervention across geographic locations, cultural contexts, and populations, prioritizing safety and promoting privacy while initiating, managing, or terminating a telehealth encounter with patients who may be at risk for or experiencing IPV. Emerging intervention guidance demonstrates ways that safety steps for virtual services are being institutionalized, for example the use of passcodes and emergency exit buttons for mobile apps, encrypted messaging, and personnel training related to screening for other people in a virtual meeting (NNEDV, 2021). Moving this work forward will have myriad benefits for survivors and service providers alike. These include logistical convenience and immediacy of the service provision, increased engagement, accessibility, and anonymity of services, and enhanced opportunities to extend services to historically underserved populations.

\section{In-person social distancing}

Findings also identified steps that could be taken to enhance agencies' ability to provide safe in-person services during future public health emergencies. First, agencies need to have on hand essential items for keeping people and spaces clean and sanitary. This includes PPE for workers and survivors, cleaning supplies, and sanitation kits, as well as considering the ability to be physically distant within residential spaces. This study identified that some agencies, such as shelters and food resources, had to continue to offer inperson services which caused advocates to be exposed to COVID-19. Offering hazard pay and additional sick leave during a future pandemic will protect advocates and prevent loss of staffing during future pandemics. Furthermore, for housing environments, having a community disaster response plan that includes emergency funding to provide extra space for housing for those living in shelters, or those needing shelter, can help agencies reduce their capacity to keep people safe. This could include resources to pay for hotel rooms, trailers or RVs, or expanded transitional housing units so that survivors in need of shelter can reduce their exposure and maintain access to safe housing. Shifts were also needed for ongoing in-person services in order to help survivors cope with social distancing while addressing experiences of interpersonal violence during the pandemic. For future public health emergencies, communal living settings could be better prepared to provide child focused programing, including offering games and supports for children so that parents can have some relief, as well as providing support and activities for adult survivors to reduce the sense of feeling trapped.

Despite the challenges in transitioning to virtual services, there is much to be gained in expanding services. Similar to efforts to expand telehealth for sexual assault survivors (Miyamoto et al., 2021; Walsh et al., 2019), virtual services may offer additional access to hard-to-reach populations and may reduce burden on clients in obtaining in-person services such as transportation and child-care costs. Emerging research examining telehealth and teletherapy approaches implemented during the pandemic indicate that clients and practitioners/service providers have adapted well to such remote services and it has allowed for consistency in service provision (Burgoyne \& Cohn, 2020; Mental Health America, 2021). Given this preliminary evidence, many are considering a continuation of such virtual services and implementing a hybrid approach that includes in-person and virtual components. Funding stemming from the Violence Against Women Act and the Victims of Crime Act could be used to support different approaches to service delivery that may consist of any combination of face-to-face and virtual services. Part of such funding could provide access to tablets or phones for those in need of such items and support the development of formal policies and guidance on providing effective, safe, and trauma-informed care in these modes. Research examining in-person, hybrid, and virtual models of domestic violence services would contribute to the evidence base on telehealth programs that are becoming a widely used method across the world (De Luca \& Calabrò, 2020).

Rapid shifts in service provision and increased workload from changes in practice approach have created risk for occupational stress for interpersonal violence services workforce, including burnout and secondary traumatic stress (STS). Before the onset of COVID-19, the victim service workforce was noted for heightened risk occupational stress due to low pay, stressful working conditions, and inconsistent organizational support (Slattery \& Goodman, 2009; Kulkarni et al 2012; Wachter et al., 2020) contributing to burnout, STS, and turnover. Early data from the pandemic suggests that the workforce is under intensified stress, and experiencing a lack of resources (Wood et al., 2020). The stress of practice shifts and increased service requests, coupled with an already stressful working environment, increases the risks for turnover. As such, agencies should use individual and relational strategies to mitigate the impact of occupational stress, such as peer support and individual counseling and organizational approaches, such as trauma-informed supervision, increased pay and leave, and supportive policies (Kulkarni et al., 2013) to reduce the 
impact of the pandemic on the workforce. Given the detrimental impact of turnover on client services (Mor Barak et al., 2001), strategies to reduce stress and help people stay in their positions positively impacts service efficacy.

\section{Limitations}

There are several limitations that should be taken into consideration when evaluating the findings and implications of this study. Although interviews were conducted anytime from three to nine months after the beginning of the pandemic response in the United States, participant contact information was collected via web-based survey within the first twelve weeks of the response. As such, service providers with greater comfort with and access to technology may have been more likely to be interviewed, as well as those who experienced less initial burden during the pandemic response. Practices will continue to shift and service providers will begin to establish new, more permanent service modes as public health restrictions are lifted and guidance changes. As such, continued work is needed to explicate the lessons of the pandemic response and understand the landscape of victims services going forward. Further, the findings are limited to voluntary victim services in the United States, and experiences may be dramatically different for workers in other settings.

\section{Conclusion}

Victim service providers faced new challenges as they adapted their services and tried to maintain life-saving connections with survivors in the wake of the COVID-19 pandemic. Based on the testimony of 33 service professionals, it is clear that the adaptability and creativity of its workforce are two of the great strengths of this service sector. Providers have met increased need and reduced in-person capacities with new ways of working, highlighting the important role that virtual services will play in the future of anti-violence work.

\section{Declarations}

Conflict of Interest The authors declare that they have no conflict of interest.

\section{References}

Badr, H., Du, H., Marshall, M., Dong, E., Squire, M., \& Gardner, L. (2020). Association between mobility patters and COVID-19 transmission in the USA: A mathematical modelling study. Lancet Infectious Disease, 20, 1247-1254.
Breiding, M. J., Smith, S. G., Basile, K. C., Walters, M. L., Chen, J., \& Merrick, M. T. (2014). Prevalence and characteristics of sexual violence, stalking, and intimate partner violence victimizationNational intimate partner and sexual violence survey, United States, 2011. Morbidity and Mortality Weekly Report: Surveillance Summaries, 63(8), 1-18.

Brignone, L., \& Edleson, J. L. (2019). The dating and domestic violence app rubric: Synthesizing clinical best practices and digital health app standards for relationship violence prevention smartphone apps. International Journal of Human-Computer Interaction, 35(19), 1859-1869.

Budinger, M. C., Cwik, M. F., \& Riddle, M. A. (2015). Awareness, attitudes, and use of crisis hotlines among youth at-risk for suicide. Suicide and Life-Threatening Behavior, 45(2), 192-198.

Burgoyne, N., \& Cohn, A. S. (2020). Lessons from the transition to relational teletherapy during COVID-19. Family Process, 59(3), 974-988. https://doi.org/10.1111/famp.12589

Castro, R., Cerellino, L., \& Rivera, R. (2017). Risk factors of violence against women in Peru. Journal of Family Violence, 32(8), 807-817. https://doi.org/10.1007/s10896-017-9929-0

Chisholm, C., Bullock, L., \& Ferguson, J. (2017). Intimate-partner violence and pregnancy: Epidemiology and impact. American Journal of Obstetrics and Gynecology, 217(2), 141-144. https:// doi.org/10.1016/j.ajog.2017.05.042

Collignon, P. (2021). COVID-19 and future pandemics: Is isolation and social distancing the new norm? International Medicine Journal, 51(5), 647-653. https://doi.org/10.1111/imj.15287

De Luca, R., \& Calabrò, R. S. (2020). How the COVID-19 pandemic is changing mental health disease management: the growing need of telecounseling in Italy. Innovations in Clinical Neuroscience, 17(4-6), 16.

Elo, S., \& Kyngas, H. (2008). The qualitative content analysis process. Journal of Advanced Nursing, 62(1), 107-115. https://doi.org/10. 1111/j.1365-2648.2007.04569.x

Jack, S. M., Munro-Kramer, M. L., Williams, J. R., Schminkey, D., Tomlinson, E., Jennings Mayo-Wilson, L., et al. (2021). Recognizing and responding to intimate partner violence using telehealth: Practical guidance for nurses and midwives. Journal of clinical nursing, 30(3-4), 588-602.

Kaukinen, C. (2020). When stay-at-home orders leave victims unsafe at home: Exploring the risk and consequences of intimate partner violence during the COVID-19 pandemic. American Journal of Criminal Justice. https://doi.org/10.1007/s12103-020-09533-5

Kulkarni, S., Bell, H., Hartman, J., \& Herman-Smith, R. (2013). Exploring individual and organizational factors contributing to compassion satisfaction, secondary traumatic stress, and burnout in domestic violence service providers. Journal of the Society for Social Work and Research, 4(2), 114-130.

Macy, R. J., Giattina, M., Sangster, T. H., Crosby, C., \& Montijo, N. J. (2009). Domestic violence and sexual assault services: Inside the black box. Aggression and Violent Behavior, 14, 359-373. https:// doi.org/10.1016/j.avb.2009.06.002

McClay, M. (2021). When 'shelter-in-place' isn't shelter that's safe: a rapid analysis of domestic violence case differences during the COVID-19 pandemic and stay-at-home orders. Journal of Family Violence, online publication, https://doi.org/10.1007/ s10896-020-00225-

Mental Health America. (2021). Teletherapy during COVID-19: What the research says. Retrieved fromhttps://mhanational.org/telet herapy-during-covid-19-what-research-says

Merolli, M., Gray, K., \& Martin-Sanchez, F. (2014). Therapeutic affordances of social media: Emergent themes from a global online survey of people with Chronic Pain. Journal of Medical Internet Research, 16(12), e284. https://doi.org/10.2196/jmir.3494

Miyamoto, S., Thiede, E., Dorn, L., Perkins, D. F., Bittner, C., \& Scanlon, D. (2021). The Sexual Assault Forensic Examination 
Telehealth (SAFE-T) Center: a comprehensive, nurse-led telehealth model to address disparities in sexual assault care. The Journal of Rural Health, 37(1), 92-102.

Mor Barak, M., Nissly, J., \& Levin, A. (2001). Antecedents to retention and turnover among child welfare, social work, and other human service employees: What can we learn from past research? A review and metanalysis. Social Service Review, 75(4), 625-661.

National Domestic Violence Hotline (NDVH). (2020). COVID-19 special report.https://www.thehotline.org/wp-content/uploads/sites/3/ 2020/06/2005-TheHot-line-COVID19-report_final.pdf

National Network to End Domestic Violence (NNEDV) (2021). Digital Services Toolkit. https://www.techsafety.org/digital-services-toolk it

Nesmith, A. (2018). Reaching young people through texting-based crisis counseling: Process, benefits, and challenges. Advances in Social Work, 18(4), 1147-1164. https://doi.org/10.18060/21590

Nnawulezi, N., Godsay, S., Sullivan, C. M., Marcus, S., \& Hacskaylo, M. (2018). The influence of low-barrier and voluntary service policies on survivor empowerment in a domestic violence housing organization. American Journal of Orthopsychiatry, 88(6), 670-680. https://doi.org/10.1037/ort0000291.

Nnawulezi, N., \& Hacskaylo, M. (2021). Identifying and responding to the complex needs of domestic violence housing practitioners at the onset of the COVID-19 pandemic. Journal of Family Violence, advance online publication, https://doi.org/10.1007/ s10896-020-00231-8

Piquero, A. R., Riddell, J. R., Bishopp, S. A., Narvey, C., Reid, J. A., \& Piquero, N. L. (2020). Staying home, staying safe? A short-term analysis of COVID-19 on Dallas domestic violence. American Journal of Criminal Justice.

Piquero, A., Jennings, W., Jemison, E., Kaukinen, C., \& Knaul, F. (2021). Domestic violence during the COVID-19 pandemicEvidence from a systematic review and meta-analysis. Journal of Criminal Justice, 74. https://doi.org/10.1016/j.jcrimjus.2021. 101806

Postmus, J. L., Plummer, S. B., McMahon, S., Murshid, N. S., \& Kim, M. S. (2012). Understanding economic abuse in the lives of survivors. Journal of Interpersonal Violence, 27, 411-430.

Rivas, C., Vigurs, C., Cameron, J., \& Yeo, L. (2019). A realist review of which advocacy interventions work for which abused women under what circumstances. Cochrane Database of Systematic Review, 6, 1-169.

Sandelowski, M. (2010). What's in a name? Qualitative description revisited. Research in Nursing \& Health., 33(1), 77-84. https:// doi.org/10.1002/nur.20362

Sedziaofa, A. P., Tenkorang, E. Y., \& Owusu, A. Y. (2016). “...he always slaps me on my ears": The health consequences of intimate partner violence among a group of patrilineal women in Ghana. Culture, Health, \& Sexuality, 18(12), 1379-1392. https://doi.org/ 10.1080/13691058.2016.1187291

Slattery, S. M., \& Goodman, L. A. (2009). Secondary traumatic stress among domestic violence advocates: Workplace risk and protective factors. Violence Against Women, 15(11), 1358-1379. https:// doi.org/10.1177/1077801209347469

Soleimani, R., Ahmadi, R., \& Yosefnezhad, A. (2017). Health consequences of intimate partner violence against married women: A population-based study in northern Iran. Psychology, Health,
\& Medicine, 22(7), 845-850. https://doi.org/10.1080/13548506. 2016.1263755

Sullivan, C. M., \& Goodman, L. A. (2019). Advocacy with survivors of intimate partner violence: What it is, what it isn't, and why it's critically important. Violence Against Women, 25(16), 2007-2023.

Taylor, C. B., Fitzsimmons-Craft, E. E., \& Graham, A. K. (2020). Digital technology can revolutionize mental health services delivery: The COVID-19 crisis as a catalyst for change. International Journal of Eating Disorders, 53(7), 1155-1157.

Tracy, S. J. (2010). Qualitative quality: Eight "big-tent" criteria for excellent qualitative research. Qualitative Inquiry, 16, 837-851. https://doi.org/10.1177/1077800410383121

United States Department of Health and Human Services (2020). COVID-19 Guidance. Retreated from https://www.hhs.gov/coron avirus/index.html

Valera, E., \& Kucyi, A. (2017). Brain injury in women experiencing intimate partner violence: Neural mechanistic evidence of invisible trauma. Brain Imaging and Behavior, 11(6), 1664-1667. https://doi.org/10.1007/s11682-016-9643-1

Walsh, W. A., Meunier-Sham, J., \& Re, C. (2019). Using telehealth for sexual assault forensic examinations: a process evaluation of a national pilot project. Journal of forensic nursing, 15(3), 152-162. https://doi.org/10.1097/JFN.0000000000000254

Wilson, J. M., Fauci, J. E., \& Goodman, L. A. (2015). Bringing traumainformed practice to domestic violence programs: A qualitative analysis of current approaches. American Journal of Orthopsychiatry, 85(6), 586-599. https://doi.org/10.1037/ort0000098

Voth Schrag, R., Hairston, D., Brown, M., \& Wood, L. (2021). Advocate and survivor perspectives on the role of technology in help seeking and services with emerging adults in higher education. Journal of Family Violence, advance online publication. https:// doi.org/10.1007/s10896-021-00279-0

Wachter, K., Voth Schrag, R., \& Wood, L. (2020). Coping behaviors mediate associations between occupational factors and compassion satisfaction among the intimate partner violence and sexual assault workforce. Journal of Family Violence, 35, 143-154.

Wood, L., Baumler, E., Voth Schrag, R., Guillot-Wright, S., Hairston, D., Temple, J., \& Torres, E. (2021a). 'Don't know where to go for help': Safety and economic needs among violence survivors during the COVID-19 pandemic. Journal of Family Violence, advance online publication. https://doi.org/10.1007/s10896-020-00240-7

Wood, L., Hairston, D., Voth Schrag, R., Clark, E., Parra-Cardona, R., \& Temple, J. (2021b) Creating a Digital Trauma Informed Space: Chat and Text Advocacy for Survivors of Violence. Journal of Interpersonal Violence, advance online publication.

Wood, L., Voth Schrag, R., Baumler, E., Hairston, D., Guillot-Wright, S., Torres, E., \& Temple, J. (2020). On the front lines of the COVID-19 pandemic: Occupational experiences of the intimate partner violence and sexual assault workforce. Journal of Interpersonal Violence, advance online publication. https://doi.org/10. 1177/0886260520983304,

Publisher's Note Springer Nature remains neutral with regard to jurisdictional claims in published maps and institutional affiliations. 